\title{
Zoo-FISH in the European mole (Talpa europaea) detects all ancestral Boreo-Eutherian human homologous chromosome associations
}

\author{
M. Volleth ${ }^{a}$ S. Müller ${ }^{b}$

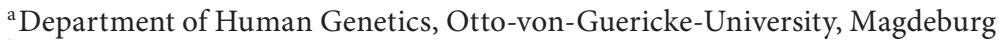 \\ ${ }^{\mathrm{b}}$ Department Biology II, Anthropology and Human Genetics, Ludwig-Maximilians-University, \\ Planegg-Martinsried (Germany)
}

Manuscript received 25 January 2006; accepted in revised form for publication by M. Schmid, 20 March 2006.

\begin{abstract}
Zoo-FISH with human whole-chromosome paint probes delineated syntenic association of human homologous chromosome segments 3-21, 14-15, 16-19, 4-8, 716 and 12-22 (twice) in the European mole (Talpa europaea, Talpidae, Eulipotyphla, Mammalia). These segment associations represent shared ancestral Boreo-Eutherian traits,
\end{abstract}

half of which were previously not described for Eulipotyphla. The karyotype of the European mole acquired a minimum of 19 translocations and six inversions compared to the presumed Boreo-Eutherian ancestor.

Copyright (C) 2006 S. Karger AG, Basel
The European mole, belonging to the family Talpidae, was formerly classified as a member of the order Insectivora, together with Soricidae (shrews), Tenrecidae (tenrecs), Solenodontidae (solenodons), Erinaceidae (hedgehogs and gymnures) and Chrysochloridae (golden moles) (MacPhee and Novacek, 1993). This order, however, has been shown to be a polyphyletic clade by DNA analyses (for references see Douady et al., 2004). After removing Afrosoricida, i.e. Chrysochloridae and Tenrecidae, from the Insectivora, a taxon called Eulipotyphla remained. Mitochondrial and nuclear gene analyses of Erinaceidae, Soricidae and Talpidae point to the monophyly of Eulipotyphla (Douady et al., 2002; Nikaido et al., 2003). According to these analyses, Eulipotyphla is the basal group of Laurasiatheria, the group further comprising Cetartiodactyla, Perissodactyla, Carnivora, Pholidota and Chiroptera (reviewed by Murphy et al., 2004).

\footnotetext{
Request reprints from Stefan Müller

Department Biology II, Anthropology and Human Genetics

Ludwig-Maximilians-University, Großhaderner Strasse 2

DE-82152 Planegg-Martinsried (Germany)

telephone: +4989 218074325; fax: +4989 218074331

e-mail: s.mueller@lrz.uni-muenchen.de
}

In contrast to the great number of reports dealing with comparative DNA analyses of Eulipotyphla, molecular cytogenetic data are only available for the common shrew, Sorex araneus (Dixkens et al., 1998). Zoo-FISH using human probes revealed that less than half of the ancestral segment combinations of Boreo-Eutherians (Froenicke, 2005) are conserved in this species (Dixkens et al., 1998). S. araneus showed shared ancestral syntenic association of human chromosomes 14-15, 3-21 and 16-19, whereas associations 4-8, 7-16 and 12-22 were not observed.

Previous analyses of differentially stained chromosomes of Talpa europaea (subspecies kratochvili), Talpa altaica and T. occidentalis indicated that the genus Talpa is karyotypically very conservative with $2 \mathrm{n}=34$ chromosomes in these three species (Zima, 1983; Jimenez et al., 1984; Kawada et al., 2002). Small differences between these mole species were observed in the amount of C-positive heterochromatin. While the European mole showed only centromeric heterochromatin (Zima, 1983), large heterochromatic segments have been found in Talpa altaica and T. occidentalis (Jimenez et al., 1984; Kawada et al., 2002).

In order to obtain more detailed insight into the chromosomal evolution of the clade Eulipotyphla we performed Zoo-FISH with human chromosome-specific painting probes to metaphase chromosomes of the European mole. 

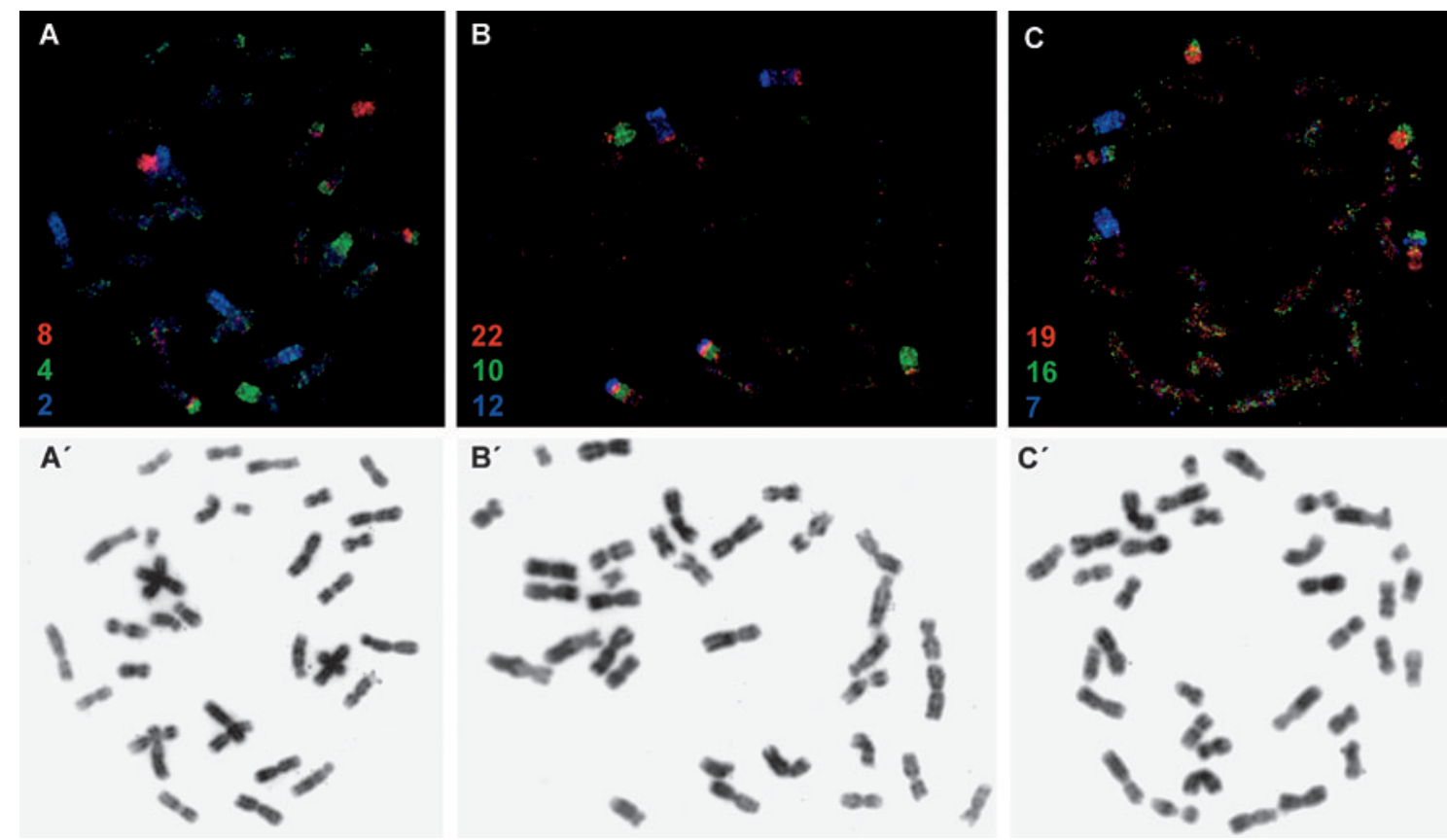

Fig. 1. Representative Zoo-FISH experiments with human chromosome-specific painting probes to metaphases of Talpa europaea delineating human homologous segment combinations proposed as ancestral for all Boreo-Eutheria. (A) Human chromosome 8 (red), 4 (green) and 2 (blue), (B) human chromosome 22 (red), 10 (green) and 12 (blue) and (C) human chromosome 19 (red), 16 (green) and 7 (blue). ( $\left.\mathbf{A}^{\prime}-\mathbf{C}^{\prime}\right)$ Respective metaphases counterstained with DAPI (inverted display).

\section{Materials and methods}

Fibroblast cultures were established from lung tissue of a deceased female mole (Talpa europaea) from North-Eastern Germany (Magdeburg). Chromosome preparation and GTG-banding were done according to standard procedures. Cross-species chromosome painting (ZooFISH) with human whole chromosome paint probes (WCPs) was essentially performed as described before (Müller et al., 1999). Briefly, human WCPs were amplified and labeled by DOP-PCR (Telenius et al., 1992) in the presence of biotin-dUTP, digoxigenin-dUTP (Roche) or Tamra-dUTP (formerly available from Applied Biosystems/PE). Two or three differentially labeled paint probes (each $0.5-1 \mu \mathrm{g}$ ) were pooled per FISH experiment, mixed with $20 \mu \mathrm{g}$ human Cot-1 DNA and hybridized to T. europaea metaphase preparations for $72 \mathrm{~h}$. Post-hybridization washes included $3 \times 5 \mathrm{~min} 2 \times \mathrm{SSC}$ at $37^{\circ} \mathrm{C}$. Biotinylated probes were detected with avidin-Cy5 (Jackson Immuno Research), digoxigeninlabeled probes with sheep-anti-digoxigenin-FITC antibody (Roche). Chromosomes were counterstained with DAPI. Metaphase images were captured with a cooled CCD camera (Photometrics C250/A equipped with a KAF1400 chip, Kodak) coupled to a Zeiss Axiophot microscope. Camera control and digital image acquisition was performed using SmartCapture VP software (Digital Scientific, Cambridge, UK).

\section{Results}

The karyotype of Talpa europaea (TEU) with $2 \mathrm{n}=34$ and $\mathrm{FN}=64$ consists of 14 large to small meta- or submetacentric and two subtelocentric autosomal pairs. The X chromosome is a medium-sized metacentric. On the short arm of TEU8 a secondary constriction is found, which has been proven to bear an active NOR by Zima (1983). CBG-banding revealed only constitutive heterochromatin in centro- meric regions (data not shown). The GTG-banding pattern is very similar to that previously described for Talpa europaea, subspecies kratochvili (Zima, 1983). It is shown in Fig. 2 together with the assignment of all hybridization signals obtained with human chromosome paint probes.

Zoo-FISH with human (HSA) WCPs to T. europaea metaphase chromosomes yielded reproducible results for all probes except for the Y. Representative FISH experiments are illustrated in Fig. 1. Altogether, the 22 human autosomes are conserved in 54 segments in T. europaea. HSA6, 9, 13, $14,17,18,20,21$ and $\mathrm{X}$ probes detected homologous segments on single T. europaea chromosomes. Only the HSA6 homologue is evolutionarily conserved as a separate chromosome in the European mole. The HSA6 synteny is however disrupted by an unpainted segment located on the proximal part of the short arm (see below). For human WCPs 2, 3, 7, 10, 12, 15, 16 and 19, hybridization signals were found on two mole chromosomes. Human WCP 5, 8 and 22 hybridized to three different $T$. europaea chromosomes, WCP 1 and 4 to four T. europaea chromosomes. In addition, human chromosomes 1, 3, 5, 16 and 19 showed homology to more than one segment of T. europaea chromosomes 3,8 , 11 and 16, indicating that these chromosomes are involved in intra-chromosomal rearrangements. Three Talpa chromosomes, TEU10, 11 and 14, bear segments homologous to five different human chromosomes. The highest number of segments per mole chromosome was found in TEU8, being composed of seven segments homologous to only two human chromosomes, i.e. 1 and 5 (Fig. 2). 
Fig. 2. G-banded karyotype of a female Talpa europaea with the assignment of the chromosome painting results for all human autosome and the X-specific WCP probes. The mole chromosomes are numbered below and the human WCPs are indicated at the right. Asterisks indicate regions on T. europaea chromosomes not hybridized by any human probe.

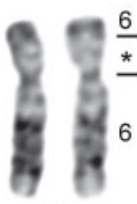

1

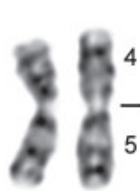

7

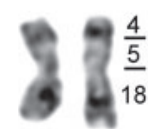

13

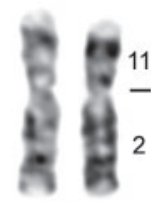

2

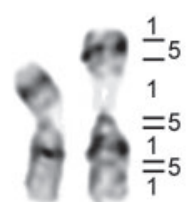

8

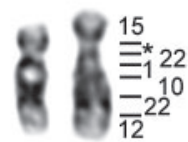

14

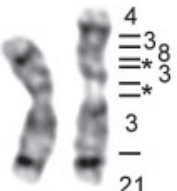

3

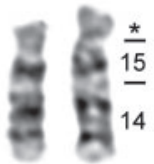

9

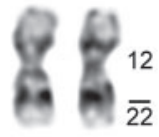

15

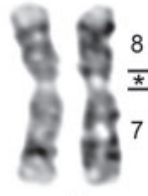

4

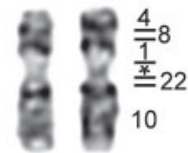

10

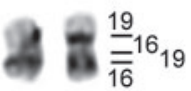

16

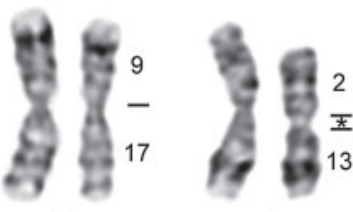

6

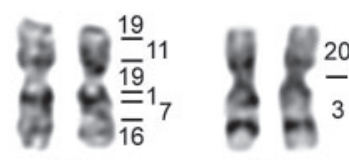

12

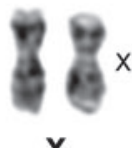

Certain segments of the Talpa europaea karyotype did not hybridize to any of the human paint probes (Fig. 2). Pericentromeric regions of chromosomes 1, 3, 4, 6, 10 and 14, the proximal part of the short arm of TEU1 and the entire short arm of TEU9 were lacking hybridization signals. Based on the knowledge of karyotype data from other members of Talpidae (Jimenez et al., 1984; Kawada et al., 2002) these segments may contain CBG-negative 'non-euchromatin' without homology to human sequences. DAPI staining revealed that these chromosome segments are not particularly AT-rich.

\section{Discussion}

The only member of Eulipotyphla for which cross-species chromosome painting data have been published up to now is Sorex araneus (Dixkens et al., 1998). Comparing this data with the Zoo-FISH results on Talpa europaea presented here, derived associations of human homologous segments 3-8 (S. araneus chromosome $3 \mathrm{q}$ and TEU3) and 7-8 (S. araneus chromosome $1 \mathrm{q}$ and TEU4) were found in both species. TEU3 (association of human 4-3-8-3-21), however, is most probably the product of a fission of the ancestral Boreo-Eutherian chromosome 4-8p-4 homologue (Froenicke, 2005, see below), followed by fusion with the 3-21 homologue and an inversion, while in $S$. araneus $1 \mathrm{q}$ association of human chromosome 3-8q homologues is found. Conversely, human 8q-7 homologues formed TEU4 while in the distal part of $S$. araneus chromosome 1 human $8 \mathrm{p}-7$ homologues are in syntenic association. In summary, associations of 7-8 and 3-8 should have formed independently in both species, hence are of different evolutionary origin and provide no phylogenetic link between Sorex and Talpa.

Shared derived syntenic association of human 8-13 homologous segments, which is present both in the common shrew and in bats may represent a phylogenetic link of Eu- lipotyphla with its sister clade Chiroptera (Volleth et al., 2002). In the European mole, however, this syntenic association was not observed. At present, it cannot be determined whether this association has been secondarily lost in the mole or if it is a convergent trait of bats and the common shrew.

Concerning segment combinations proposed as ancestral for all Boreo-Eutheria (Froenicke, 2005 for recent review), three out of seven have been found in Sorex: association of homologues to human chromosome 3-21, 14-15 and 16-19. Our results show that these syntenic associations are also present in the European mole. Moreover, the ancestral Boreo-Eutherian associations 4-8 and 7-16 are present in T. europaea and hence in the karyotype of the putative ancestral Eulipotyphla. The remaining two ancestral BoreoEutherian associations contain homologous sequences to human chromosomes 12-22 (twice). Both are also present in the European mole, one of it in combination with a segment homologous to human chromosome 10. The association 10-12-22 is also present in some other Boreo-Eutherians (Froenicke, 2005 and references therein). On TEU14, however, the smaller 12-22 homologous segment, and not the larger 12-22 element as in the other species investigated, is found together with HSA10 homologous sequences. The segment combination 10-12-22 present on European mole chromosome 14 may therefore either be the result of a species-specific fusion. Alternatively, it could be speculated that this segment combination may represent a state that is very close to the ancestral condition of Eutheria, when further considering observations on chromosome 4 of the golden mole (Chrysochloris asiaticus, Afrotheria) (Robinson et al., 2004). Chrysochloris chromosome 4 consists of homologous sequences to human chromosomes 10p, 12pq, 22qter in the long arm and 22qprox and 12qter in the short arm (Robinson et al., 2004). Supposing this chromosome form as ancestral, a centric fission would create the $10 \mathrm{p}$ 12pq-22qter element found in carnivores and Afrotheria. 
The situation found in the mole could be interpreted as the result of a pericentric inversion of the $10 \mathrm{p}$ segment and subsequent centric fission, followed by additional rearrangements.

Despite the evolutionary conservation of the above-mentioned ancestral Boreo-Eutherian chromosome forms, the karyotype of the European mole is clearly derived. It differs from the proposed ancestral Boreo-Eutherian karyotype by at least 19 translocations and six inversions. At present all chromosomal characters found in the mole have to be considered as autapomorphic traits.
By conclusion, these experiments provided answers to a number of open questions concerning the presence of shared ancestral Boreo-Eutherian chromosome forms in Eulipotyphla. Moreover, our results further substantiate the validity of the recently reconstructed ancestral Boreo-Eutherian karyotype with $2 \mathrm{n}=46$ chromosomes (Froenicke, 2005). We expect that further Zoo-FISH studies of shrews and hedgehogs will be important complementary tools to current comparative DNA analyses in the effort to shed light on Eulipotyphla phylogenomic relationships.

\section{References}

Dixkens C, Klett C, Bruch J, Kollak A, Serov OL, et al: Zoo-FISH analysis in insectivores: 'Evolution extols the virtue of the status quo'. Cytogenet Cell Genet 80:61-67 (1998).

Douady CJ, Chatelier PI, Madsen O, De Jong WW, Catzeflies F, et al: Molecular phylogenetic evidence confirming the Eulipotyphla concept and in support of hedgehogs as the sister group to shrews. Mol Phylogenet Evol 25:200-209 (2002).

Douady CJ, Scally M, Springer MS, Stanhope MJ: 'Lipotyphlan' phylogeny based on the growth hormone receptor gene: a reanalysis. Mol Phylogenet Evol 30:778-788 (2004).

Froenicke L: Origins of primate chromosomes - as delineated by Zoo-FISH and alignments of human and mouse draft genome sequences. Cytogenet Genome Res 108:122-138 (2005).

Jimenez R, Burgos M, Dias del la Guardia R: Karyotype and chromosome banding in the mole (Talpa occidentalis) from the south-east of the Iberian Peninsula. Implications on its taxonomic position. Caryologia 37:253-258 (1984).

Kawada S, Harada M, Grafodatsky AS, Oda S: Cytogenetic study of the Siberian mole, Talpa altaica (Insectivora: Talpidae) and karyological relationships within the genus Talpa. Mammalia 66:53-62 (2002).

MacPhee RDE, Novacek MJ: Definition and relationships of Lipotyphla, in Szalay FS, Novacek MJ, McKenna MC (eds): Mammal Phylogeny: Placentals, Vol. 2, pp 13-31 (Springer, New York 1993)

Müller S, Stanyon R, O'Brien PC, Ferguson-Smith MA, Plesker R, Wienberg J: Defining the ancestral karyotype of all primates by multidirectional chromosome painting between tree shrews, lemurs and humans. Chromosoma 108: 393-400 (1999).
Murphy WJ, Pevzner WA, O’Brien SJ: Mammalian phylogenomics comes of age. Trends Genet 20 631-638 (2004)

Nikaido M, Cao Y, Harada M, Okada N, Hasegawa M: Mitochondrial phylogeny of hedgehogs and monophyly of Eulipotyphla. Mol Phylogenet Evol 28:276-284 (2003).

Robinson TJ, Fu B, Ferguson-Smith MA, Yang F: Cross-species chromosome painting in the golden mole and elephant-shrew: support for the mammalian clades Afrotheria and Afroinsectiphillia but not Afroinsectivora. Proc R Soc Lond B 271:1477-1484 (2004).

Telenius H, Pelmear A, Tunnacliffe A, Carter NP, Behmel A, et al: Cytogenetic analysis by chromosome painting using DOP-PCR amplified flow-sorted chromosomes. Genes Chromosomes Cancer 4:257-263 (1992).

Volleth M, Heller KG, Pfeiffer RA, Hameister H: A comparative ZOO-FISH analysis in bats elucidates the phylogenetic relationships between Megachiroptera and five microchiropteran families. Chromosome Res 10:477-497 (2002).

Yang F, Graphodatsky AS, Li T, Fu B, Dobigny G, et al: Comparative genome maps of the pangolin, hedgehog, sloth, anteater and human revealed by cross-species chromosome painting: further insight into the ancestral karyotype and genome evolution of eutherian mammals. Chromosome Res 14:283-296 (2006).

Ye J, Biltueva L, Huang L, Nie W, Wang J, et al: Cross-species chromosome painting unveils cytogenetic signatures for the Eulipotyphla and evidence for the polyphyly of Insectivora. Chromosome Res 14:151-159 (2006).

Zima J: The karyotype of Talpa europaea kratochvili (Talpidae, Insectivora). Folia Zoologica 32: 131-136 (1983)

\section{Note added in proof}

Since this report was accepted for publication, comparative chromosome maps between human and three additional Eulipotyphla were published (Yang et al., 2006; Ye et al., 2006). From the data obtained on the longeared hedgehog (Hemiechinus auritus), the short-tailed shrew (Blarinella griselda) and the shrew-hedgehog (Neotetracus sinensis), Ye et al. (2006) conclude that syntenic segment association of human 4-20 homologues represents a lineage-specific chromosome signature for Eulipotyphla. This association, however, was not found in the mole (this study), but in the Javan pangolin (Manis javanica, Pholidota; Yang et al., 2006). We consider the association $4-5$ as a reliable Eulipotyphla signature since it is present in all species studied so far with the exception of the highly rearranged karyotype of $S$. araneus. 